\title{
Antenna Elevation Control using Multiple Switched Self- Tuning Controllers Design
}

\author{
Ahmed M. Alnajeh ${ }^{1 *}$, Othman E. Aburas ${ }^{2}$, Youssef Amer Arebi ${ }^{3}$ \\ ${ }^{1}$ Department of Electrical and Computer Engineering, Applied Research and Development \\ Center, Tripoli, Libya. \\ ${ }^{2}$ Department of Electrical and Computer Engineering, Faculty of Engineering Alkhoms, \\ Elmergib University, Libya. \\ ${ }^{3}$ Department of Electrical and Computer Engineering, Advanced Center of Technology, \\ Tripoli, Libya.
}

DOI: https://doi.org/10.21467/proceedings.2.26

* Corresponding author email: aalnajeh@yahoo.com

\begin{abstract}
Adaptive controllers have a lot of advantages over conventional ones, especially when the model of the plant to be controlled is unknown or changes with time. This paper proposes a control scheme for multiple adaptive Self-Tuning Pole-Placement controllers using both the classical technique via transfer function and the modern technique using discrete state-space. This approach enables the user to switch between the classical and modern techniques in order to control the estimated plant model on-line; the switching mechanism ensures a smooth transition amongst the two pole-placement controllers.

The performance of the proposed control scheme on the closed-loop performance of an antenna system, controlling its elevation, is demonstrated. Simulation results demonstrating the effectiveness of the switching mechanism between different controllers are presented. A Graphical User Interface is built to facilitate the controller programming and allowing the simulation of multiple adaptive controllers.
\end{abstract}

Keywords: Adaptive Self-Tuning Control, Discrete State-Space, Pole-Placement Control, Antenna Elevation Control.

\section{Introduction}

Control systems design techniques typically require an in-depth understanding of the plant under study and its environment. In some applications, however, the plant to be controlled is sophisticated and the involved physical processes are changeable with time and operating conditions. To deal with such situations, different approaches of adaptive control are proposed to tune the controller parameters and behavior in response to the physical processes changes [1]. Self-tuning controllers represent an important class of adaptive control since they provides systematic and flexible approaches for dealing with many difficulties including time varying parameters, non-linearity, and uncertainties. Recently, there has been increasing interest in pole-placement self-tuning controllers due to the fact that in the regulator case, they provide mechanisms to overcome the restriction to minimum phase plants of some optimal

(C) 2018 Copyright held by the author(s). Published by AIJR Publisher in Proceedings of First Conference for Engineering

Sciences and Technology (CEST-2018), September 25-27, 2018, vol. 1 .
This is an open access article under Creative Commons Attribution-NonCommercial 4.0 International (CC BY-NC 4.0)

This is an open access article under Creative Commons Attribution-NonCommercial 4.0 International (CC BY-NC 4.0)
license, which permits any non-commercial use, distribution, adaptation, and reproduction in any medium, as long as the
original work is properly cited. ISBN: 978-81-936820-5-0 
Antenna Elevation Control using Multiple Switched Self-Tuning Controllers Design

controllers. In the servo case, they give the ability to directly introduce the natural angular frequency $\omega_{n}$ and damping ratio $\zeta$ as tuning parameters. Moreover, robustness is an essential advantage of pole-placement methods, as they simply modify the system dynamics instead of cancelling them as applied in optimal self-tuning controller [2]. However, the main drawbacks of self-tuning pole-placement controller are based on transfer function approach and also their inability to regulate steady-state error in the presence of constant disturbances. The transfer function approach depends on polynomial algorithms, which are slow to emerge [3]. In contrast, the linear algebraic tools that are required by state-space techniques are a lot more advanced and more suitable for optimal control design [1][4]. Therefore, the state-space technique is preferred over the transfer function approach, especially for multivariable and non-linear systems [5]. The main contribution of this paper is to develop a control scheme for multiple adaptive Self-Tuning Pole-Placement controllers using both the classical technique via transfer function and the modern technique using discrete state-space framework. In order to assess the performance of the proposed scheme, it is applied to single-input-single-output of an antenna model.

\section{A Servomechanism for an Antenna Elevation Control}

It is desired to control the elevation of an antenna designed to track a geostationary satellite as sketched in Figure 1. The antenna and drive parts have a moment of inertia $\mathbf{J}^{\prime}$ and damping $\mathrm{B}_{\mathrm{r}}$ arising to some extent from bearings and aerodynamic friction, but mostly from the back emf $(\mathrm{V})$ of the DC-drive motor $[6,7,8]$.

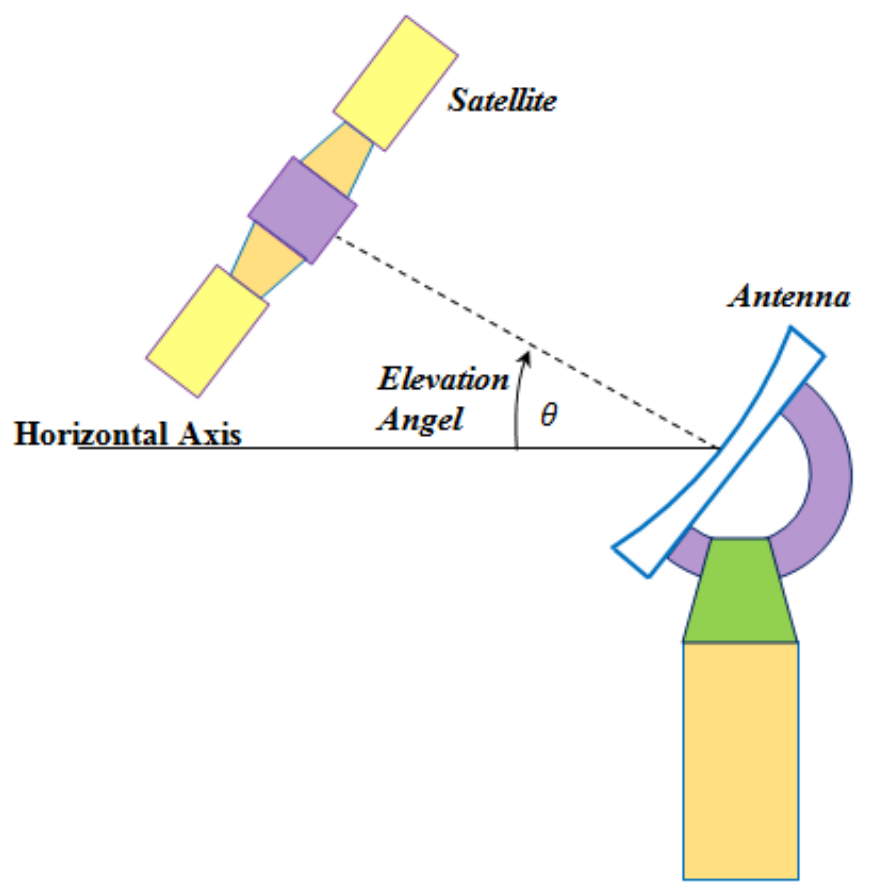

Figure 1: Schematic Diagram of Antenna System 
Figure 2 shows the internal connection of DC-drive motor with the antenna system. Here, the armature inductance $L_{a}(H)$ is negligible because it is usually small. The continuous transfer function [7], [8] can be given by:

$\theta(s)=\frac{a}{s(s+a)}\left[U(s)+\xi^{\prime}(s)\right]$

where $\xi^{\prime}(s)$ is the torque disturbance due to wind, and $U(s)$ is the torque due to the DC motor.

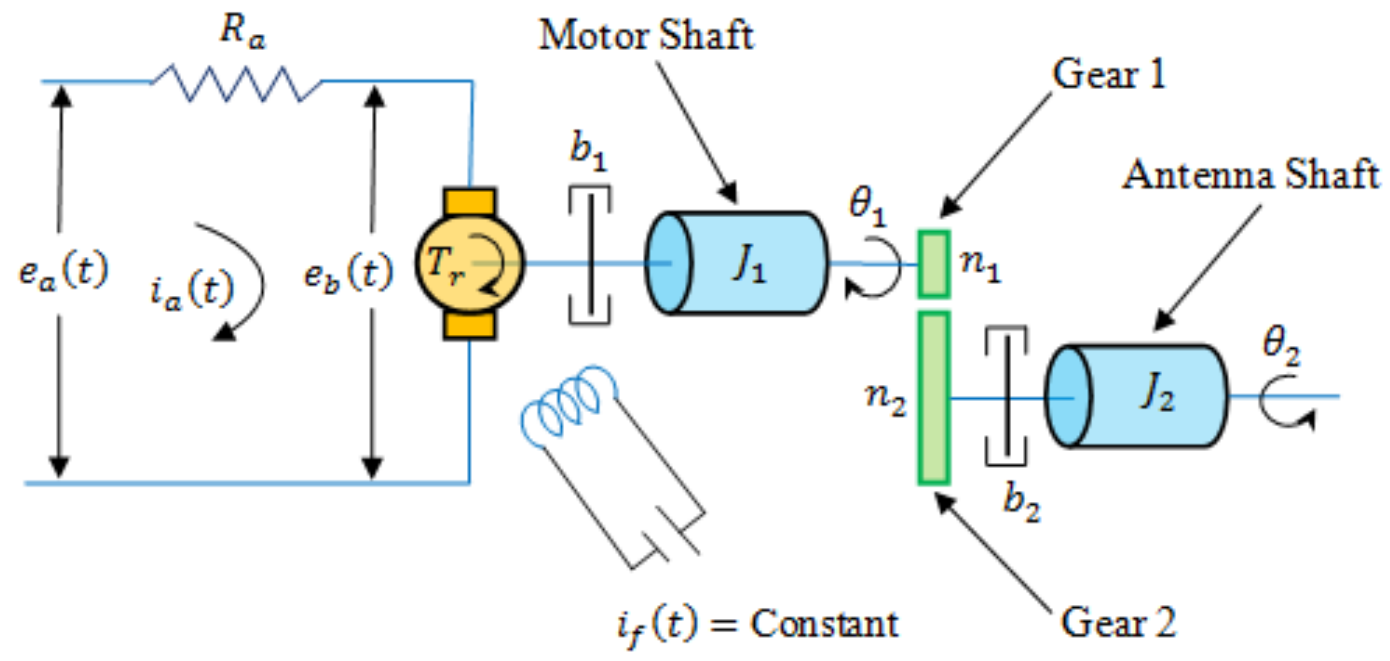

Figure 2: The Connection of DC-drive Motor with the Antenna System

The aim of the design is to measure the error between the angle of the satellite $\theta_{s}(t)(\mathrm{rad})$ and the antenna $\theta(t)(\mathrm{rad})$ and compute $u(t)$ so that the error $e$, i.e. equals to $\left(\theta_{s}(t)-\right.$ $\theta(t))(\mathrm{rad})$, is always less than $0.001 \mathrm{rad}$ during tracking. The geostationary satellite angle that must be followed can be adequately approximated by a fixed velocity.

$$
\theta_{s}(t)=(0.01 \mathrm{rad} / \mathrm{sec}) \times t(\mathrm{sec}) .
$$

The discrete model of the Antenna system can be written as:

$\theta(z)=\frac{\left(a T_{s}-1+e^{-a T_{s}}\right) z+\left(1-e^{-a T_{s}}-a T_{s} e^{-a T_{s}}\right)}{a(z-1)\left(z-e^{-a T_{s}}\right)}\left[U(z)+\xi^{\prime}(z)\right]$

A discrete state-space [9] of antenna tracking control model in which the time constant $\mathrm{a}=$ 0.1 , and $T_{s}=1 \mathrm{sec}$ is:

$\left[\begin{array}{l}X_{1}(t+1) \\ X_{2}(t+1)\end{array}\right]=\left[\begin{array}{cc}0 & 1 \\ -0.9048 & 1.905\end{array}\right]\left[\begin{array}{l}X_{1}(t) \\ X_{2}(t)\end{array}\right]+\left[\begin{array}{l}0.04837 \\ 0.13895\end{array}\right] u(t)+\left[\begin{array}{l}0.04837 \\ 0.13895\end{array}\right] \xi^{\prime}$

$y(t)=\left[\begin{array}{ll}1 & 0\end{array}\right]\left[\begin{array}{l}X_{1}(t) \\ X_{2}(t)\end{array}\right]$

In (3), $X_{1}(t)$ is the position (rad)and $X_{2}(t)$ is the velocity ( $\left.\mathrm{rad} / \mathrm{sec}\right)$ of the antenna. 
Antenna Elevation Control using Multiple Switched Self-Tuning Controllers Design

\section{Adaptive Control Algorithm}

The Controlled Auto-Regressive Moving Average (CARMA) process model [10] is described as:

$A\left(z^{-1}\right) y(t)=z^{-k} B\left(z^{-1}\right) u(t)+C\left(z^{-1}\right) \xi^{\prime}(t)$

Assume that the polynomials $A\left(z^{-1}\right)$ and $B\left(z^{-1}\right)$ are co-prime, i.e. they do not have any common factors. Furthermore, $A\left(z^{-1}\right), C\left(z^{-1}\right)$ are monic, i.e. the coefficient of the highest power is unity [11]. The classical pole-placement controller can be described by the following control-law:

$q\left(z^{-1}\right) u(t)=H\left(z^{-1}\right) r(t)-F\left(z^{-1}\right) y(t)$

where $q\left(z^{-1}\right), F\left(z^{-1}\right)$ and $H\left(z^{-1}\right)$ are polynomials in the back shift operator $z^{-1}$.

The controller has two degrees of freedom, the first is a feed forward with the transfer operator $\frac{H\left(z^{-1}\right)}{q\left(z^{-1}\right)}$ and the second is a feedback with the transfer operator $\frac{F\left(z^{-1}\right)}{q\left(z^{-1}\right)}$. A block diagram of the closed-loop system is shown in Figure 3. The controller polynomials $H\left(z^{-1}\right)$, $F\left(z^{-1}\right)$ and $q\left(z^{-1}\right)$ are designed to ensure fast output tracking of the reference signal $r(t)$.

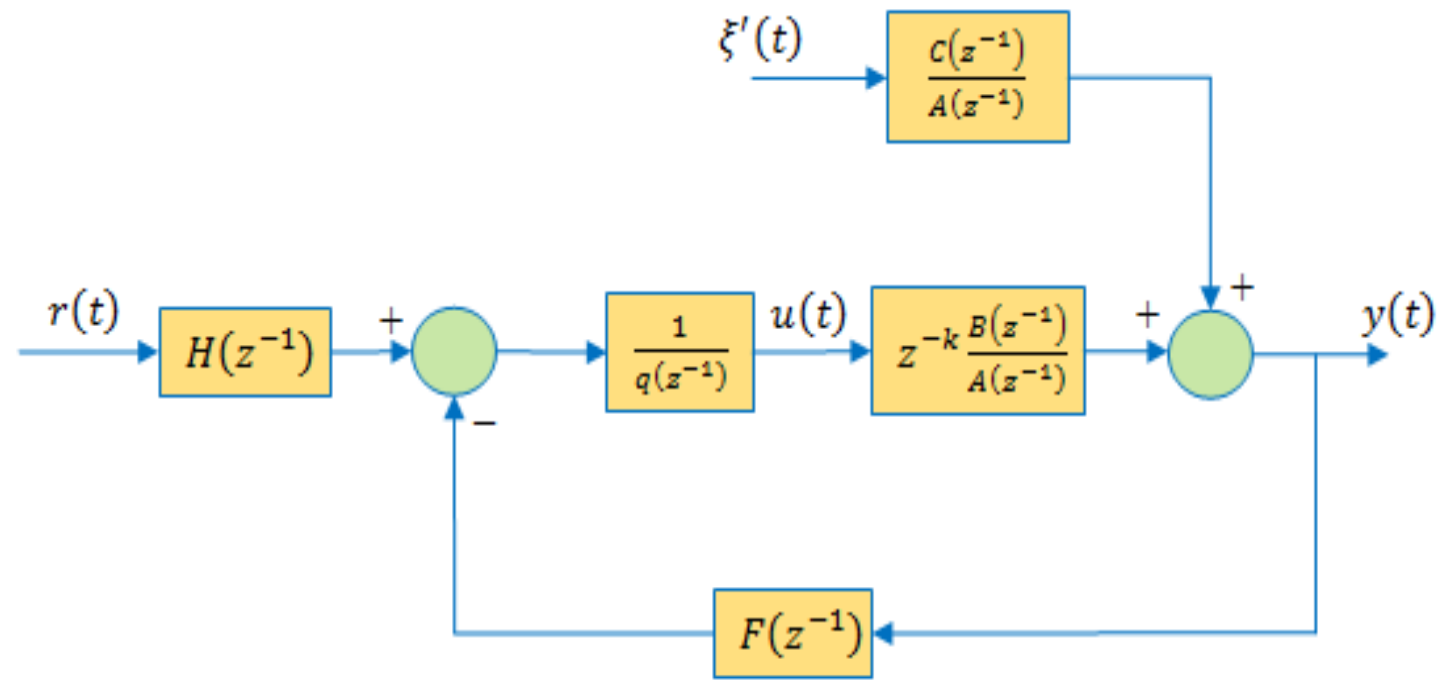

Figure 3: Classical Discrete Pole-Placement Controller

The closed-loop characteristic polynomial of the system (Diophantine equation) is [4]:

$$
q\left(z^{-1}\right) A\left(z^{-1}\right)+z^{-k} B\left(z^{-1}\right) F\left(z^{-1}\right)=T_{c}\left(z^{-1}\right) C\left(z^{-1}\right)
$$

The main concept of the pole placement controller design is to specify the desired closed-loop poles polynomial $\boldsymbol{T}_{\boldsymbol{c}}\left(\boldsymbol{z}^{-\mathbf{1}}\right)$ as a design parameter. By solving the Diophantine equation (7), the polynomials $\boldsymbol{q}\left(\mathbf{z}^{-\mathbf{1}}\right)$ and $\boldsymbol{F}\left(\mathbf{z}^{-\mathbf{1}}\right)$ can be obtained. The closed-loop poles polynomial 
$\overline{\boldsymbol{T}_{\boldsymbol{c}}\left(\mathbf{z}^{-\mathbf{1}}\right) \text { fundamentally determines the property and the performance of the closed system }}$ [11].

The desired closed loop poles polynomial $\boldsymbol{T}_{\boldsymbol{c}}\left(\mathbf{z}^{\mathbf{- 1}}\right)$ and the controller parameters polynomials $\boldsymbol{F}\left(\mathbf{z}^{-\mathbf{1}}\right)$ and $\boldsymbol{q}\left(\mathbf{z}^{-\mathbf{1}}\right)$ are expressed in terms of $\mathbf{z}^{-\mathbf{1}}$ as follows:

$$
F\left(z^{-1}\right)=f_{0}+f_{1} z^{-1}+\cdots+f_{n_{a}-1} z^{-n_{f}+1}+f_{n_{f}} z^{-n_{f}}
$$

$$
q\left(z^{-1}\right)=1+q_{1} z^{-1}+\cdots+q_{n_{q}-1} z^{-n_{q}+1}+q_{n_{q}} z^{-n_{q}}
$$

$$
T_{c}\left(z^{-1}\right)=1+t_{1} z^{-1}+\cdots+t_{n_{t}-1} z^{-n_{t}+1}+t_{n_{t}} z^{-n_{t}}
$$

where, the parameters $\boldsymbol{t}_{\mathbf{1}}$ and $\boldsymbol{t}_{\mathbf{2}}$ are specified as following [11]:

$$
\begin{gathered}
t_{1}=-2 \exp \left(-\zeta \omega_{n} T_{s}\right) \cos \left(T_{s} \omega_{n} \sqrt{1-\zeta^{2}}\right) \\
t_{2}=-2 \exp \left(-\zeta \omega_{n} T_{s}\right)
\end{gathered}
$$

Where $\boldsymbol{\zeta}$ and $\boldsymbol{\omega}_{\boldsymbol{n}}$ are respectively the damping ratio and natural angular frequency of the second order closed loop transient response and $\boldsymbol{T}_{\boldsymbol{s}}$ is the sampling time. In order to have a unique solution, the polynomials $\boldsymbol{F}\left(\mathbf{z}^{-\mathbf{1}}\right), \boldsymbol{q}\left(\mathbf{z}^{-\mathbf{1}}\right)$ and $\boldsymbol{T}_{\boldsymbol{c}}\left(\mathbf{z}^{-\mathbf{1}}\right)$ in the equations (8), (9), and (10) are specified as follows:

$$
\left.\begin{array}{l}
n_{q}=n_{b}+k-1 \\
n_{f}=n_{a}-1 \\
n_{t} \leq n_{a}+n_{b}+k-n_{c}-1
\end{array}\right\}
$$

Substituting Diophantine equation (7) into equation (6), the following equation is obtained:

$y(t)=\frac{z^{-k} B\left(z^{-1}\right) H\left(z^{-1}\right)}{T_{c}\left(z^{-1}\right) C\left(z^{-1}\right)} r(t)+\frac{q\left(z^{-1}\right)}{T_{c}\left(z^{-1}\right)} \xi^{\prime}(t)$

It can be seen from equation (12) that the closed loop poles are placed at their pre-specified positions given by the desired closed loop poles polynomial $\boldsymbol{T}_{\boldsymbol{c}}\left(\boldsymbol{z}^{-\mathbf{1}}\right)$ which represents the design parameter. The controller algorithm explained above can be structured as a self-tuning controller as shown in Figure 4. Where, all of the controller's parameters are calculated depending on the change in plant parameters.

Proceedings of First Conference for Engineering Sciences and Technology (CEST-2018), vol. 1 


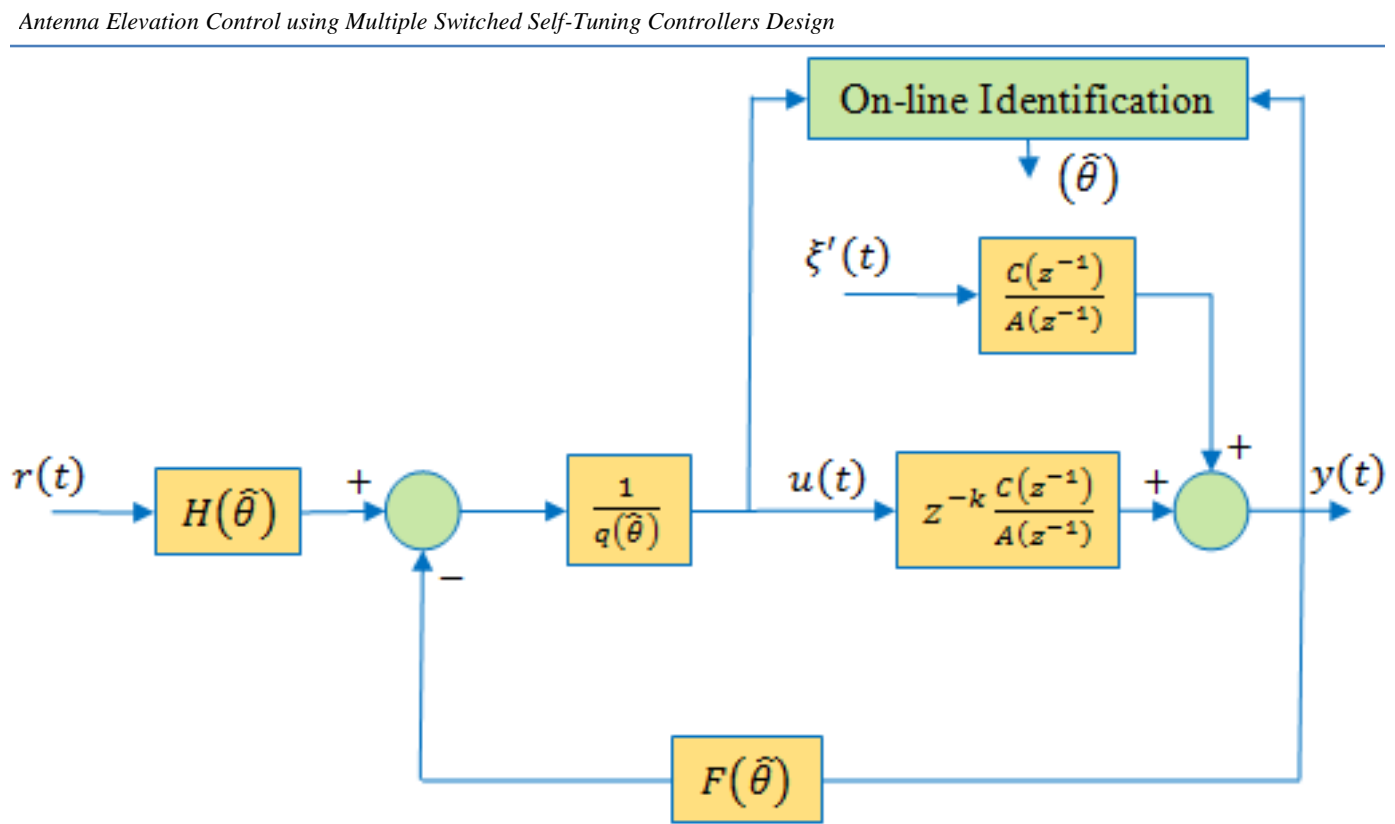

Figure 4: Classical Self-Tuning Pole-Placement Controller

The modern Self-Tuning Pole-Placement using discrete state-space control design algorithm is given in Figure 5, which is based on an on-line observer with a state feedback [6].

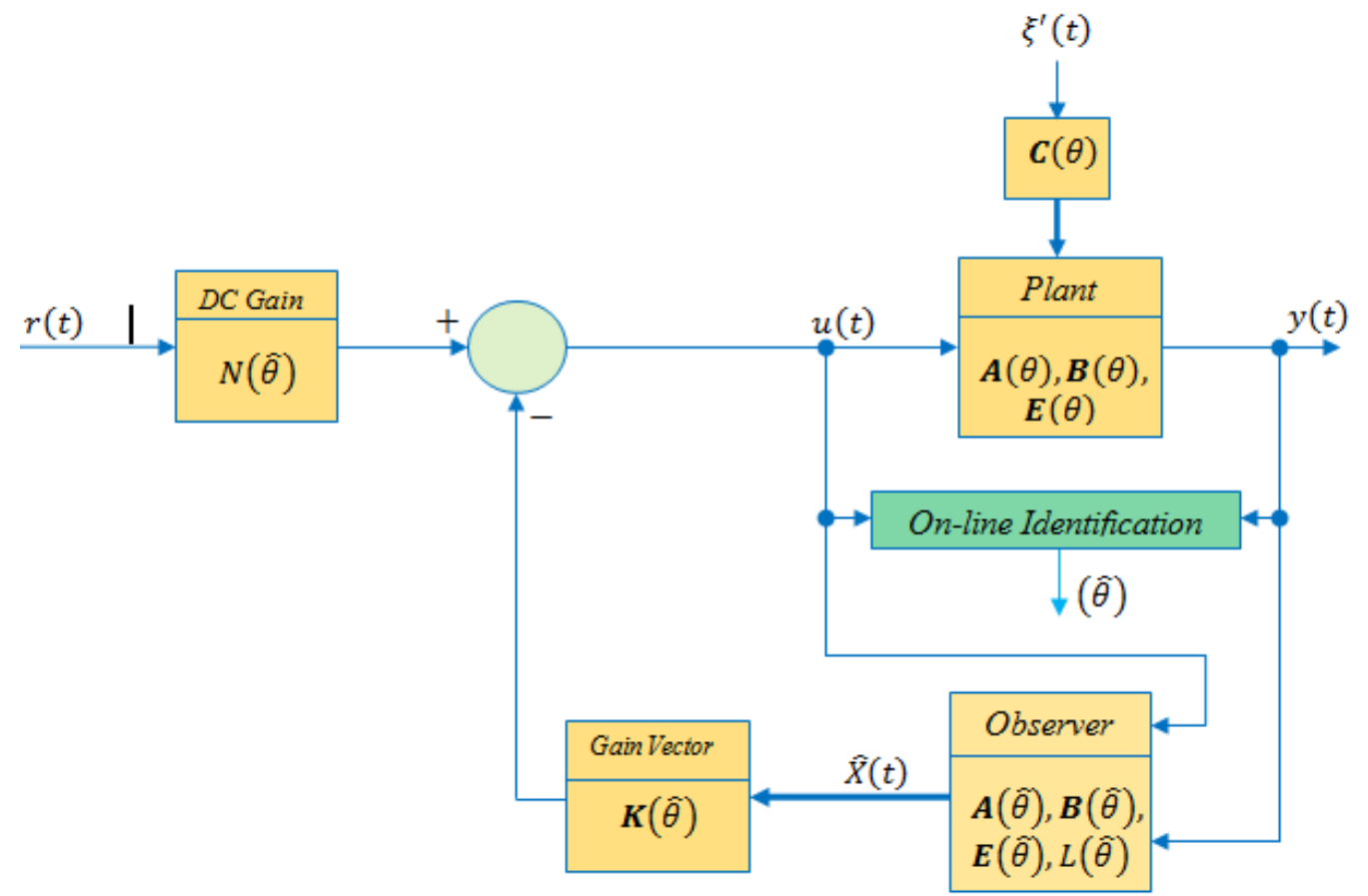

Figure 5: Shows the modern Self-Tuning pole-placement controller.

Both an on-line observer poles and the closed-loop system poles are placed based on the model parameters $(\widehat{\boldsymbol{\theta}})$ obtained from the on-line identification scheme (RLS or ERLS 
estimators). In Figure 5, the proper dc gain $\mathbf{N}(\widehat{\boldsymbol{\theta}})$ is introduced on-line into the design in the presence of reference signal $\mathbf{r}(\mathbf{t})$ to eliminate the output steady state error. A discrete statespace model of any system can be derived and presented in discrete matrix-vector equation as follows:

$X(t+1)=A X(t)+B u(t)+C \xi^{\prime}(t)$

$y(t)=E X(t)+b_{0} u(t)+\xi^{\prime}(t)$

The values of both control input signal $\mathbf{u}(\mathbf{t})$ and system output signal $\mathbf{y}(\mathbf{t})$ are read for every sampling instant; these values are used for on-line identification methods such as (RLS or ERLS estimators). An on-line identification method can be used to estimate plant parameters $\widehat{\boldsymbol{\theta}}$ which are then used to identify state-space model as:

$\boldsymbol{X}(\boldsymbol{t}+\mathbf{1})=\boldsymbol{A}(\widehat{\boldsymbol{\theta}}) \boldsymbol{X}(\boldsymbol{t})+\boldsymbol{B}(\widehat{\boldsymbol{\theta}}) \boldsymbol{u}(\boldsymbol{t})+\boldsymbol{C}(\widehat{\boldsymbol{\theta}}) \xi^{\prime}(\boldsymbol{t})$

$$
\boldsymbol{y}(\boldsymbol{t})=\boldsymbol{E}(\widehat{\boldsymbol{\theta}}) \boldsymbol{X}(\boldsymbol{t})
$$

\section{(16)}

where, the estimated plant parameters $\widehat{\boldsymbol{\theta}}=\left[-\widehat{\boldsymbol{a}}_{\mathbf{1}}-\widehat{\boldsymbol{a}}_{\mathbf{2}} \cdots-\right.$

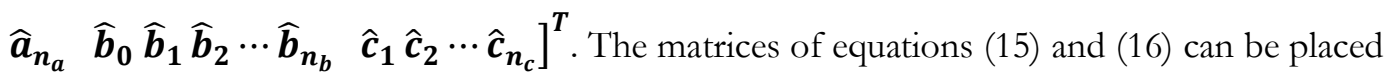
in either plant framework or observer framework [7].

The transparent controllable canonical form is illustrated as follows:

$$
\boldsymbol{X}(\boldsymbol{t}+\mathbf{1})=\boldsymbol{A}_{c}(\widehat{\boldsymbol{\theta}}) \boldsymbol{X}(\boldsymbol{t})+\boldsymbol{B}_{c}(\widehat{\boldsymbol{\theta}}) \boldsymbol{u}(\boldsymbol{t})+\boldsymbol{C}_{c}(\widehat{\boldsymbol{\theta}}) \xi^{\prime}(\boldsymbol{t})
$$

$$
\begin{aligned}
& \text { (17) } \\
& \boldsymbol{y}(\boldsymbol{t})=\boldsymbol{E}_{\boldsymbol{c}}(\widehat{\boldsymbol{\theta}}) \boldsymbol{X}(\boldsymbol{t}) \\
& \text { where: } \\
& \boldsymbol{A}_{c}(\widehat{\boldsymbol{\theta}})=\left[\begin{array}{ccccc}
\mathbf{0} & \mathbf{1} & \mathbf{0} & \cdots & \mathbf{0} \\
\mathbf{0} & \mathbf{0} & \mathbf{1} & \cdots & \mathbf{0} \\
\vdots & \vdots & \vdots & & \vdots \\
\mathbf{0} & \mathbf{0} & \mathbf{0} & & \mathbf{1} \\
-\widehat{\boldsymbol{a}}_{\boldsymbol{n}_{a}} & -\widehat{\boldsymbol{a}}_{\boldsymbol{n}_{a}-1} & -\widehat{\boldsymbol{a}}_{\boldsymbol{n}_{a}-2} & \cdots & -\widehat{\boldsymbol{a}}_{1}
\end{array}\right], \boldsymbol{B}_{c}(\widehat{\boldsymbol{\theta}})=\left[\begin{array}{c}
\mathbf{0} \\
\mathbf{0} \\
\vdots \\
\mathbf{1}
\end{array}\right], \\
& \boldsymbol{E}_{\boldsymbol{c}}(\widehat{\boldsymbol{\theta}})=\left[\begin{array}{llll}
\widehat{\boldsymbol{b}}_{\boldsymbol{n}_{\boldsymbol{b}}} & \widehat{\boldsymbol{b}}_{\boldsymbol{n}_{\boldsymbol{b}}-\mathbf{1}} & \cdots & \widehat{\boldsymbol{b}}_{\mathbf{1}}
\end{array}\right] \text { and } \boldsymbol{C}_{\boldsymbol{c}}(\widehat{\boldsymbol{\theta}})=\left[\begin{array}{llll}
\widehat{\boldsymbol{c}}_{\boldsymbol{n}_{\boldsymbol{c}}} & \widehat{\boldsymbol{c}}_{\boldsymbol{n}_{\boldsymbol{c}}-\mathbf{1}} & \cdots & \widehat{\boldsymbol{c}}_{\mathbf{1}}
\end{array}\right]
\end{aligned}
$$

The transparent observable canonical form can be represented as:

$$
X(t+1)=A_{o}(\widehat{\theta}) X(t)+B_{o}(\widehat{\theta}) u(t)+C_{o}(\widehat{\theta}) \xi^{\prime}(t)
$$

$$
y(t)=E_{o}(\widehat{\theta}) X(t)
$$




\section{Where}

$$
\begin{gathered}
\boldsymbol{A}_{\boldsymbol{o}}(\widehat{\boldsymbol{\theta}})=\left[\begin{array}{ccccc}
\mathbf{0} & \mathbf{0} & \cdots & \mathbf{0} & -\widehat{\boldsymbol{a}}_{\boldsymbol{n}_{a}} \\
\mathbf{1} & \mathbf{0} & \cdots & \mathbf{0} & -\widehat{\boldsymbol{a}}_{\boldsymbol{n}_{a}-\mathbf{1}} \\
\vdots & \vdots & & & \vdots \\
\mathbf{0} & \mathbf{0} & \cdots & \mathbf{1} & -\widehat{\boldsymbol{a}}_{1}
\end{array}\right], \boldsymbol{B}_{o}(\widehat{\boldsymbol{\theta}})=\left[\begin{array}{c}
\widehat{\boldsymbol{b}}_{\boldsymbol{n}_{b}} \\
\widehat{\boldsymbol{b}}_{\boldsymbol{n}_{b}-\mathbf{1}} \\
\vdots \\
\widehat{\boldsymbol{b}}_{1}
\end{array}\right], \boldsymbol{C}_{\boldsymbol{o}}(\widehat{\boldsymbol{\theta}})=\left[\begin{array}{c}
\widehat{\boldsymbol{c}}_{\boldsymbol{n}_{c}} \\
\widehat{\boldsymbol{c}}_{\boldsymbol{n}_{b}-\mathbf{1}} \\
\vdots \\
\widehat{\boldsymbol{c}}_{1}
\end{array}\right] \text { and } \\
E_{o}(\widehat{\boldsymbol{\theta}})=\left[\begin{array}{lllll}
\mathbf{0} & \mathbf{0} & \cdots & \mathbf{0} & \mathbf{1}
\end{array}\right]
\end{gathered}
$$

The on-line controller design in discrete state-space based on one framework allows calculating a matrix $\boldsymbol{T}(\widehat{\boldsymbol{\theta}})$ that transforms between canonical frameworks, which is given as:

$$
\begin{gathered}
\boldsymbol{R}_{c}(\widehat{\boldsymbol{\theta}})=\left[\begin{array}{lllll}
\boldsymbol{E}_{c}(\widehat{\boldsymbol{\theta}}) & \boldsymbol{E}_{c}(\widehat{\boldsymbol{\theta}}) \boldsymbol{A}_{c}(\widehat{\boldsymbol{\theta}}) & \cdots & \boldsymbol{E}_{c}(\widehat{\boldsymbol{\theta}}) \boldsymbol{A}_{c}^{\boldsymbol{n}-\mathbf{1}}(\widehat{\boldsymbol{\theta}})
\end{array}\right] \\
\mathbf{R}_{\mathbf{0}}^{-\mathbf{1}}(\widehat{\boldsymbol{\theta}})=\left[\begin{array}{ccccc}
\hat{\mathbf{a}}_{1} & \hat{\mathbf{a}}_{\mathbf{2}} & \cdots & \hat{\mathbf{a}}_{\mathbf{n}-\mathbf{1}} & \mathbf{1} \\
\hat{\mathbf{a}}_{\mathbf{2}} & \cdots & \hat{\mathbf{a}}_{\mathbf{n}-\mathbf{1}} & \mathbf{1} & \mathbf{0} \\
\vdots & \vdots & \mathbf{1} & \mathbf{0} & \mathbf{0} \\
\hat{\mathbf{a}}_{\mathbf{n}-\mathbf{1}} & \mathbf{1} & \mathbf{0} & \mathbf{0} & \mathbf{0} \\
\mathbf{1} & \mathbf{0} & \mathbf{0} & \mathbf{0} & \mathbf{0}
\end{array}\right], \quad \mathbf{T}(\widehat{\boldsymbol{\theta}})=\mathbf{R}_{\mathbf{o}}^{-\mathbf{1}}(\widehat{\boldsymbol{\theta}}) \times \mathbf{R}_{\mathbf{c}}(\widehat{\boldsymbol{\theta}})
\end{gathered}
$$

where, $\mathbf{T}(\widehat{\boldsymbol{\theta}})$ is the transformation matrix between transparent canonical frameworks, $\mathbf{R}_{\mathbf{c}}(\widehat{\boldsymbol{\theta}})$ is the observability matrix of transparent controllable canonical form and $\mathbf{R}_{\mathbf{o}}^{-\mathbf{1}}(\widehat{\boldsymbol{\theta}})$ is the inverse observability matrix of transparent observable canonical form.

The estimated state vector $\widehat{\mathbf{X}}_{\mathbf{c}}(\mathbf{t})$ of an on-line observer of transparent controllable canonical form (plane framework) can be evaluated as follows:

$$
\widehat{\boldsymbol{X}}_{c}(\boldsymbol{t}+\mathbf{1})=\boldsymbol{A}_{c}(\widehat{\boldsymbol{\theta}}) \widehat{\boldsymbol{X}}_{c}(\boldsymbol{t})+\boldsymbol{L}_{c}(\widehat{\boldsymbol{\theta}})\left(\boldsymbol{y}(\boldsymbol{t})-\boldsymbol{E}_{c}(\widehat{\boldsymbol{\theta}}) \widehat{\boldsymbol{X}}_{c}(\boldsymbol{t})\right)+\boldsymbol{B}_{c}(\widehat{\boldsymbol{\theta}}) \boldsymbol{u}(\boldsymbol{t})
$$

The on-line observer gain matrix $\mathbf{L}(\widehat{\boldsymbol{\theta}})$ can be easily calculated by using transparent observable canonical form [7], therefore, the on-line observer gains $\mathbf{L}(\widehat{\boldsymbol{\theta}})$ is transformed to transparent controllable canonical form to be used in equation (22) as follows:

$$
L_{c}(\widehat{\theta})=T \times L_{o}(\widehat{\theta})=T \times\left[\begin{array}{c}
L_{o_{1}}(\widehat{\theta}) \\
L_{o_{2}}(\widehat{\theta}) \\
\vdots \\
L_{o_{n}}(\widehat{\theta})
\end{array}\right]=T \times\left[\begin{array}{c}
\widehat{a}_{n_{a}}-\sigma_{o(n)} \\
\widehat{a}_{n_{a}-1}-\sigma_{o(n-1)} \\
\vdots \\
\widehat{a}_{1}-\sigma_{o(1)}
\end{array}\right]=\left[\begin{array}{c}
L_{c_{1}}(\widehat{\theta}) \\
L_{c_{2}}(\widehat{\theta}) \\
\vdots \\
L_{c_{n}}(\widehat{\theta})
\end{array}\right]
$$

The equation of the transparent controllable canonical form gain $\boldsymbol{K}_{\boldsymbol{c}}(\widehat{\boldsymbol{\theta}})$ can be written as:

$$
\begin{aligned}
& K_{c}(\widehat{\boldsymbol{\theta}})=\left[\begin{array}{lllll}
K_{c_{1}}(\widehat{\boldsymbol{\theta}}) & , & K_{c_{2}}(\widehat{\boldsymbol{\theta}}) & \cdots & \boldsymbol{K}_{\boldsymbol{c}_{\boldsymbol{n}}}(\widehat{\boldsymbol{\theta}})
\end{array}\right] \\
& =\left[\begin{array}{llll}
\widehat{a}_{n_{a}}-\alpha_{c(n)} & , \quad \widehat{a}_{n_{a}-1}-\alpha_{c(n-1)} & \cdots & \widehat{a}_{1}-\alpha_{c(1)}
\end{array}\right]
\end{aligned}
$$

The proper dc gain $\boldsymbol{N}$ can be calculated as follows: 


\section{$(N(\widehat{\theta}))^{-1}=-E_{c}(\widehat{\theta})\left(A_{c}(\widehat{\theta})-B_{c}(\widehat{\theta}) K_{c}(\widehat{\theta})-I\right)^{-1} B_{c}(\widehat{\theta})$}

Referring to Figure (7), the control-law can be generated as follows:

$$
\boldsymbol{u}(\boldsymbol{t})=\boldsymbol{N}(\widehat{\boldsymbol{\theta}}) \boldsymbol{r}(\boldsymbol{t})-\boldsymbol{K}_{\boldsymbol{c}}(\widehat{\boldsymbol{\theta}}) \widehat{\boldsymbol{X}}_{c}(\boldsymbol{t})
$$

The algorithm of Self-Tuning Observer Pole-placement with Reference Signal and Proper DC Gain can be summarized as follows:

Step 1: Select the desired control-low characteristic equation $\boldsymbol{\alpha}_{\mathbf{c}}(\mathbf{z})$ and the desired observer characteristic equation $\boldsymbol{\sigma}_{\mathbf{o}}(\mathbf{z})$.

Step 2: Read the new values of $\mathbf{y}(\mathbf{t})$ and $\mathbf{u}(\mathbf{t})$.

Step 3: Estimate the process parameters $\widehat{\boldsymbol{\theta}}$ using recursive least squares estimator or extended recursive least squares estimator and formulate a state-space model of the plant $\left\{\mathbf{A}_{\mathbf{c}}(\widehat{\boldsymbol{\theta}}), \mathbf{B}_{\mathbf{c}}(\widehat{\boldsymbol{\theta}}), \mathbf{E}_{\mathbf{c}}(\widehat{\boldsymbol{\theta}}), \mathbf{C}_{\mathbf{c}}(\widehat{\boldsymbol{\theta}})\right\}$ using equations (17) and (18).

Step 4: Evaluate $\mathbf{L}_{\mathbf{c}}(\widehat{\boldsymbol{\theta}})$ using equation (23).

Step 5: Estimate the state vector $\widehat{\mathbf{X}}_{\mathbf{c}}(\mathbf{t})$ using equation (22).

Step 6: Calculate $\mathbf{K}_{\mathbf{c}}(\widehat{\boldsymbol{\theta}})$ using equation (24).

Step 7: Compute $\mathbf{N}(\widehat{\boldsymbol{\theta}})$ using equation (25).

Step 8: Apply the control input signal using equation (26).

Step 2 to 8 are repeated for every sampling instant.

Both the modern self-tuning pole-placement, shown in Figure 6, and the classical controllers were programmed as multiple controller algorithms. The design provides a choice of using either classical or modern pole-placement controller on-line throughout the flick of switches $\left(\mathbf{S}_{\mathbf{1}}, \mathbf{S}_{\mathbf{2}}\right)$. The switching (transition) decision between these different fixed structure controllers is achieved manually in order to demonstrate the feasibility of the proposed approach. Each control mode can be switched on with the flick of the switches depending on the user's choice, whereas the other controller is at standby. This design also, provides the

Proceedings of First Conference for Engineering Sciences and Technology (CEST-2018), vol. 1 
Antenna Elevation Control using Multiple Switched Self-Tuning Controllers Design

possibility to choose an on-line identification method such recursive least squares (RLS) or extended recursive least squares (ERLS) estimators.

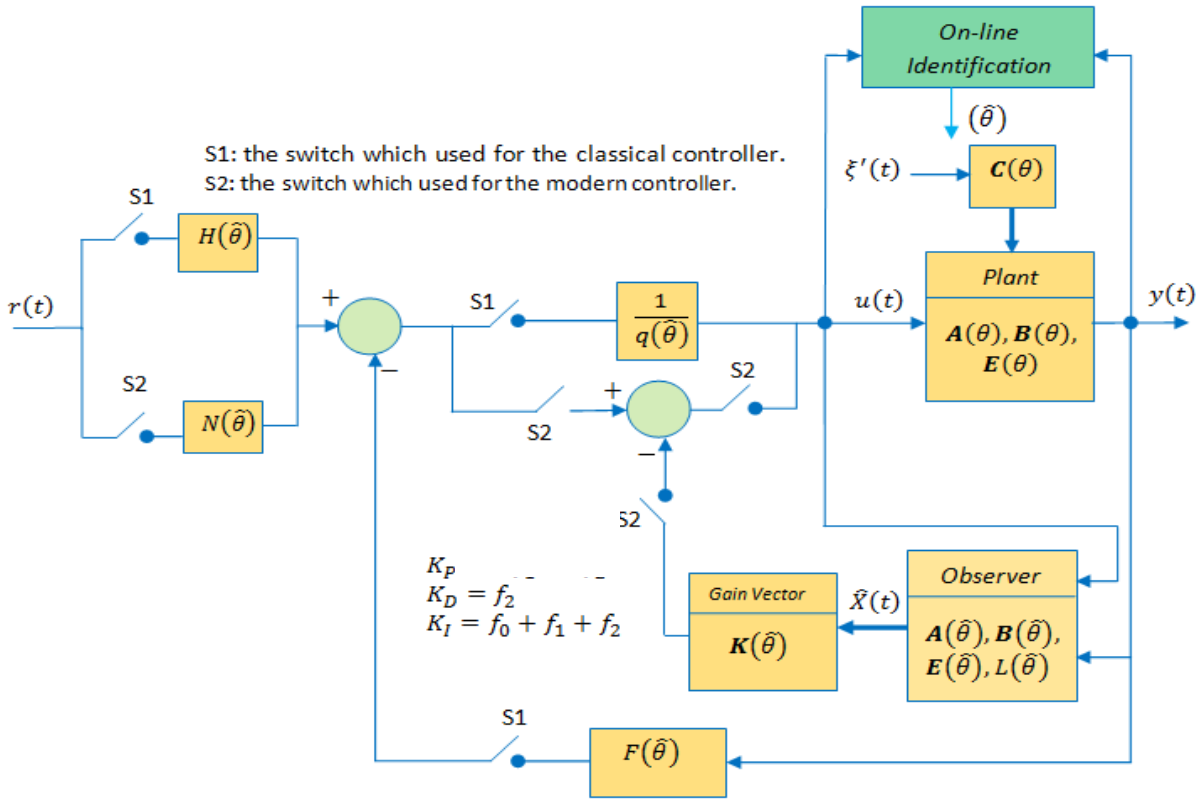

Figure 6: Multiple Self-tuning Controllers

\section{Simulation Results}

The main aim of developing a GUI is to simplify the control algorithms discussed in previous section so that the simulations can be carried out by users who do not even have a previous knowledge about the algorithms of self-tuning controllers. Thus, controller tuning and evaluation of the closed-loop performance can be realized interactively using the GUI in a user-friendly environment as shown in Figure 7.

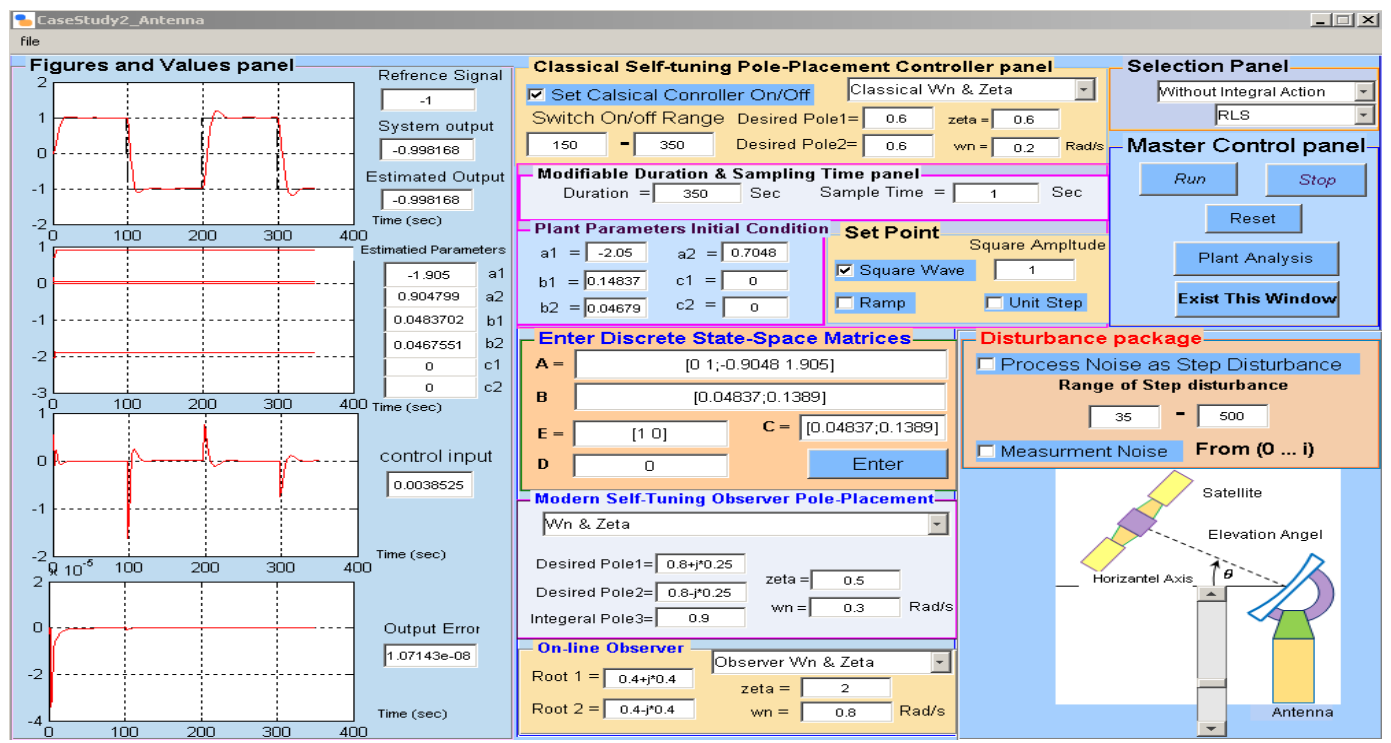

Figure 7: Multiple Controllers GUI 
To study the response of the antenna output using multiple controllers, a simulation was carried out using the system described by the discrete state-space equations (8) and (9). The simulation was performed using recursive least squares estimator over 350 samples with a sample time of 1s (approximately 6 minutes) to track a rectangular signal (in dotted black line). The desired set point is a square wave signal that has peak values of 1 and -1 with a duration of 100 samples. The signal and the response using the Modern Self-Tuning Observer PolePlacement controller is shown in the first 150 sample instants in Figure 8-a. The response of the system using the Classical Self-Tuning Pole-Placement controller is used after the $150^{\text {th }}$ sample. The control input for the two Ressgese are shown in Figure 8-b. Figures 8-a shows that these controllers are matched without any transient behaviour during switching mode.

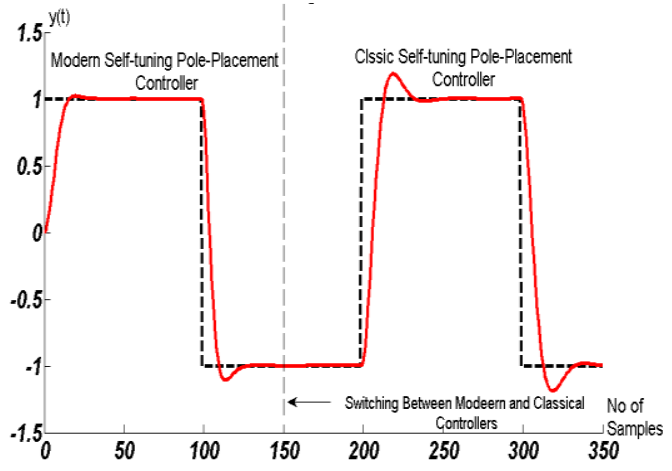

(a)

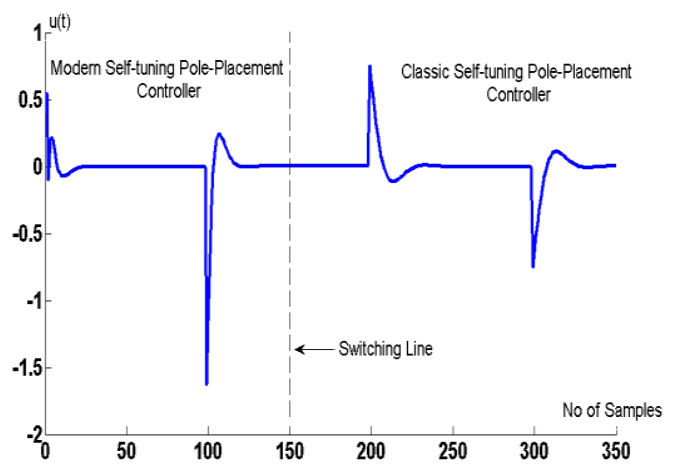

(b)

Figure 8: (a) Antenna outputs response for multiple controllers, (b) Multiple control input to the Antenna system

Another simulation was performed over 400 samples (approximately 7 minutes) using recursive least squares (RLS) estimator to track a triangular signal changes from 1 to 0 and from 0 to 1 every 100 samples instants. In this simulation, the classical self-tuning pole placement controller was switched on at 150th sampling instant, whereas the modern STOPPRI controller was used in first part of intervals as shown in Figure 8 and Figure 9.

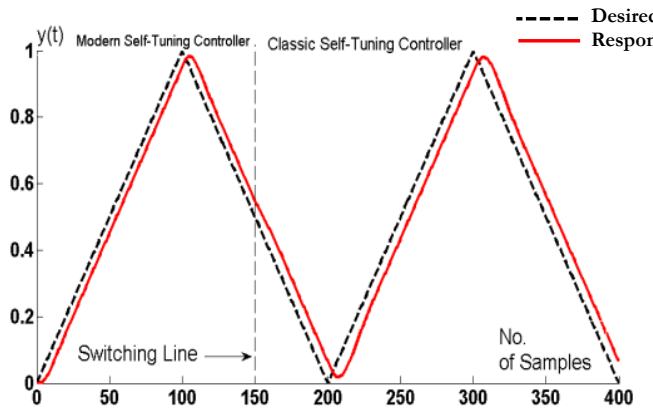

(a)

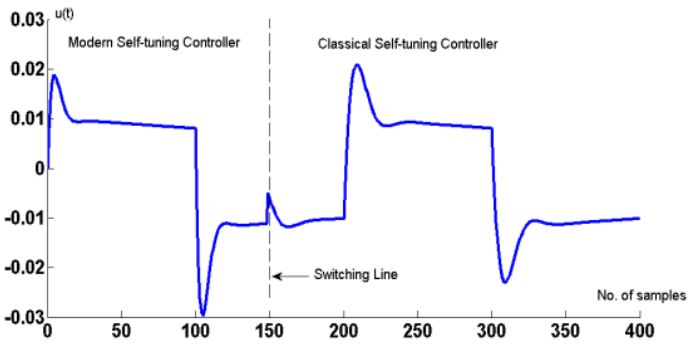

(b)

Figure 9: (a) Antenna System Output using modern and classical Controllers (b) Control Input of the Antenna System using classical and modern adaptive Controllers 
Antenna Elevation Control using Multiple Switched Self-Tuning Controllers Design

In Figure 9-b, a small transient behaviour appeared at the 150th sampling instant during switching between modern and classical self-tuning controllers which does not affect the antenna response as shown in Figure 9-a and disappeared at steady-state region. 5Conclusions A multiple controller scheme incorporating an adaptive mechanism using classical transfer function technique and modern discrete state-space technique was designed the scheme was simulated with the application to antenna model controlling its elevation. This scheme enables the user to effectively switch between the classical and modern controllers. Once the desired controller is selected to be on-line, the other controller remains standby to ensure robust control performance in the presence of controller failure. Simulation testing the proposed method were carried out and shows the performance of the proposed technique.

\section{Acknowledgment}

The authors would like to thank Ausama Ahmed for his help in writing the manuscript.

\section{References}

[1] A. Karl and B. Wittenmark, Adaptive Control, Courier Corporation, 2013.

[2] D. W. Clarke, C. Mohtadi and P. S. Tuffs, "Generalized predictive control—Part I. The basic algorithm," Automatica, vol. 23, no. 2, pp. 137-148, 1987.

[3] Z. Gao, "Scaling and bandwidth-parameterization based controller tuning," in Proceedings of the American control conference\}, 2006.

[4] Narendra KS, Cheng Xiang, 2000, 'Adaptive Control of Discrete-time Systems using Multiple Models', IEEE Transaction on Automatic Control, 45, 1669-1686.

[5] P. N. Paraskevopoulos, 2002, 'Modern control Engineering', CRC Press Taylor \& Francis Group.

[6] Gene F. Franklin, J. David Powell, Michael L. Workman, 1998, 'Digital Control of Dynamic Systems', $3^{\text {nd }}$ ed., Addison-Wesley.

[7] Katsuhiko Ogata, 1992, 'System Dynamics', $2^{\text {nd }}$ ed., Prentice Hall.

[8] Norman S. Nise, 2004, 'Control System Engineering ', $4^{\text {th }}$ ed., John Wiley \& Sons.

[9] M. Gopal, 2003, "Digital Control and State Variable Methods", $2^{\text {nd }}$ ed., Prentice Hall.

[10] Zhongshan Wu, 2001, 'Simulation Study And Instability Of Adaptive Control', MSc., The Department of Electrical and Computer Engineering, Louisiana State University, Louisiana, USA.

[11] P. E. Wellstead, M. B. Zarrop, 1991, 'Self-Tuning Systems: Control and Signal Processing', John Wiley \& Sons. 